\title{
Congenital pulmonary airway malformation
}

\author{
Khanbabaee $\mathrm{G}^{1}$, Tabatabaei $\mathrm{SA}^{1}$, Rahimpoor $\mathrm{F}^{2}$, Khatami $\mathrm{A}^{3}$, Jadali $\mathrm{F}^{4}$, Ghoroobi $\mathrm{J}^{5}$, Sheibani $\mathrm{K}^{6}$, Rezaei $\mathrm{N}^{7,8}$ \\ Department of Pediatric Respiratory Diseases, Mofid Children Hospital, Shahid Beheshti Medical University of Medical Sciences \\ Tehran,Iran. khanbabaeegh@yahoo.com
}

\begin{abstract}
Background: Congenital cystic adenomatoid malformations (CCAMs) are considered rare developmental anomalies of the lower respiratory tract. These are hamartomatous abnormalities of the lung with adenomatoid proliferation of cysts resembling bronchioles and usually occur sporadically and unilaterally with single lobe involvement.

Method: A 6-year-old girl was admitted to our center because of prolonged fever and non-productive cough lasting3 months before admission.

Results: The only other complaint was night sweating. She did not have dyspnea and did not mention any respiratory symptoms. On examination, coarse crackle and decreased lung sounds in the left side were detected. White blood cell count was $9.100 / \mu \mathrm{L}$, hemoglobin was $11.2 \mathrm{~g} / \mathrm{dL}$, erythrocyte sedimentation rate was 50 and Creactive protein was $1+. \operatorname{IgA}$ and IgM for hydatid cyst were tested and both were raised (14 and 1.4, respectively). Conclusion: The patient underwent surgery, with the probable diagnosis of hydatid cyst but in operating room diagnosis was changed and it was adenomatoid cystic malformation. In follow-up, she was in good general condition without any post-surgical complaints (Fig. 3, Ref. 11). Full Text in PDF www.elis.sk.

Key words: cystic adenomatoid malformation of lung, congenital, pulmonary, pneumonectomy.
\end{abstract}

Congenitalcystic adenomatoidmalformations(CCAMs)areconsidered rare developmental anomalies of the lower respiratory tract (1). These lesions are also called congenital pulmonary airway malformations (CPAMs), due to some types being non-cystic. CCAMs are non-hereditary, hamartomatous lung abnormalities with adenomatoid proliferation of cysts resembling bronchioles and usually occur sporadically and unilaterally with single lobe involvement (2).

CCAM's formation is not related to factors such as race, age or exposure (1), but occurs more often in males with a ratio of 1.8 to 1 and associated anomalies are rare (3).

Data from large population registries suggest an incidence of congenital lung cysts in the range of 1 per 8,300 to 35,000 live births. Large cyst subtypes account for about 70 percent of CCAM incidence (1).

Herein, a girl with history of prolonged fever and non-productive cough is presented in whom the diagnosis of CCAM was confirmed.

${ }^{1}$ Department of Pediatric Respiratory Diseases, Mofid Children Hospital, Shahid Beheshti Medical University of Medical Sciences, Tehran, Iran, ${ }^{2}$ Department of Pediatrics, Mofid Children Hospital, Shahid Beheshti Medical University of Medical Sciences, Tehran, Iran, ${ }^{3}$ Department of Radiology, Mofid Children Hospital, Shahid Beheshti Medical University of Medical Sciences, Tehran, Iran, ${ }^{4}$ Surgical Ward, Mofid Children Hospital, Shahid Beheshti Medical University of Medical Sciences, Tehran, Iran, ${ }^{5}$ Department of Pathology, Mofid Children Hospital, Shahid Beheshti Medical University of Medical Sciences, Tehran, Iran, ${ }^{6} \mathrm{Clinical}$ Research and Development Center, Imam Hossein Medical Center, Shahid Beheshti Medical University of Medical Sciences, Tehran, Iran, ${ }^{7}$ Research Center for Immunodeficiencies, Pediatrics Center of Excellence, Children's Medical Center, Tehran University of Medical Sciences, Tehran, Iran, and ${ }^{8}$ Molecular Immunology Research Center; and Department of Immunology, School of Medicine, Tehran University of Medical Sciences, Tehran, Iran Address for correspondence: G. Khanbabaee, MD, Department of Pediatric Respiratory Diseases, MofidChildren's Hospital, ShariatiAvenue, Tehran, Iran. Phone: +9821.2222.70208, Fax: +9821.2222.7033

\section{Case report}

A 6-year-old girl was admitted to the Mofid Children Hospital, Tehran, Iran because of prolonged fever and non-productive cough lasting 3 months before admission. She was referred from Lorestan province. The continuous cough would cause her to vomit and become cyanotic. The other complaint was night sweating. She did not have dyspnea and any respiratory symptoms before and this was the first time she experienced such symptoms. On examination, coarse crackle and decreased lung sounds in the left side were detected. After antibiotic administration the temperature reached to $37{ }^{\circ} \mathrm{C}$ with respiratory rate of $18 /$ minute, pulse rate of $90 /$ minute and blood pressure of $110 / 70 \mathrm{mmHg}$.

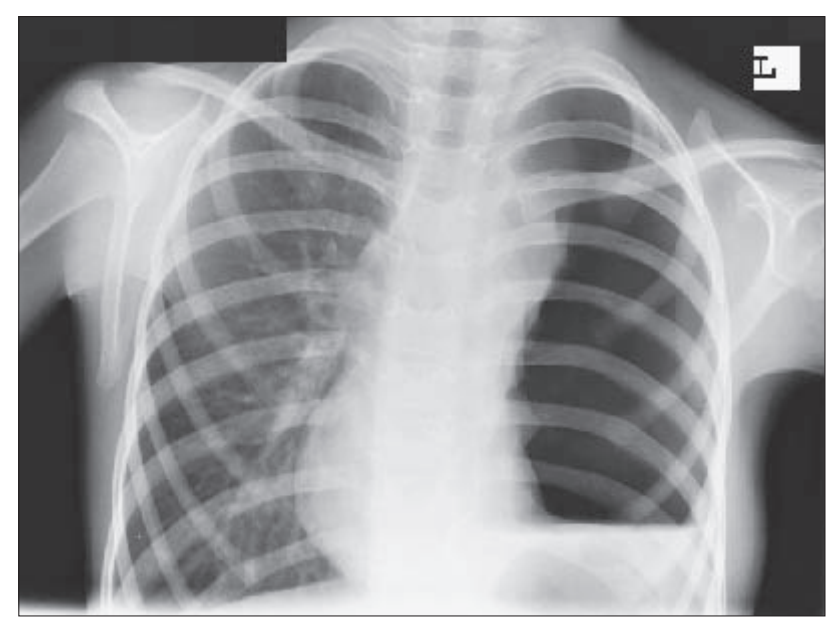

Fig. 1. Left-sided hyper-aeration and left lung collapse on chest $X$-ray. 

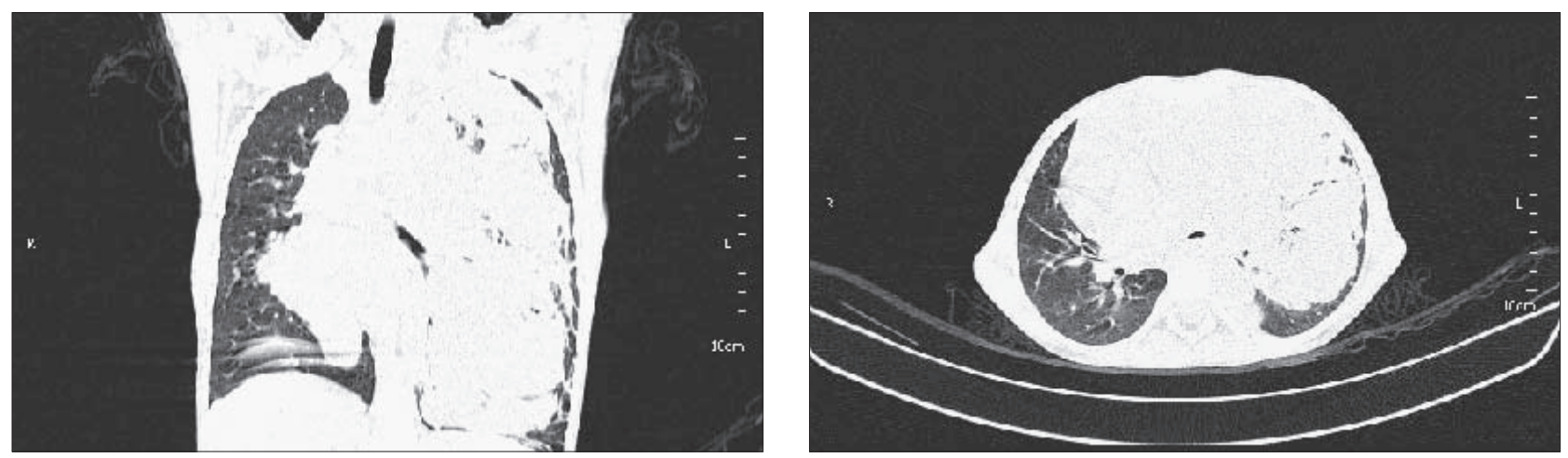

Fig. 2. Multiloculated cystic lesion in left hemithorax with mass effect and midline shift on computed tomography scan.
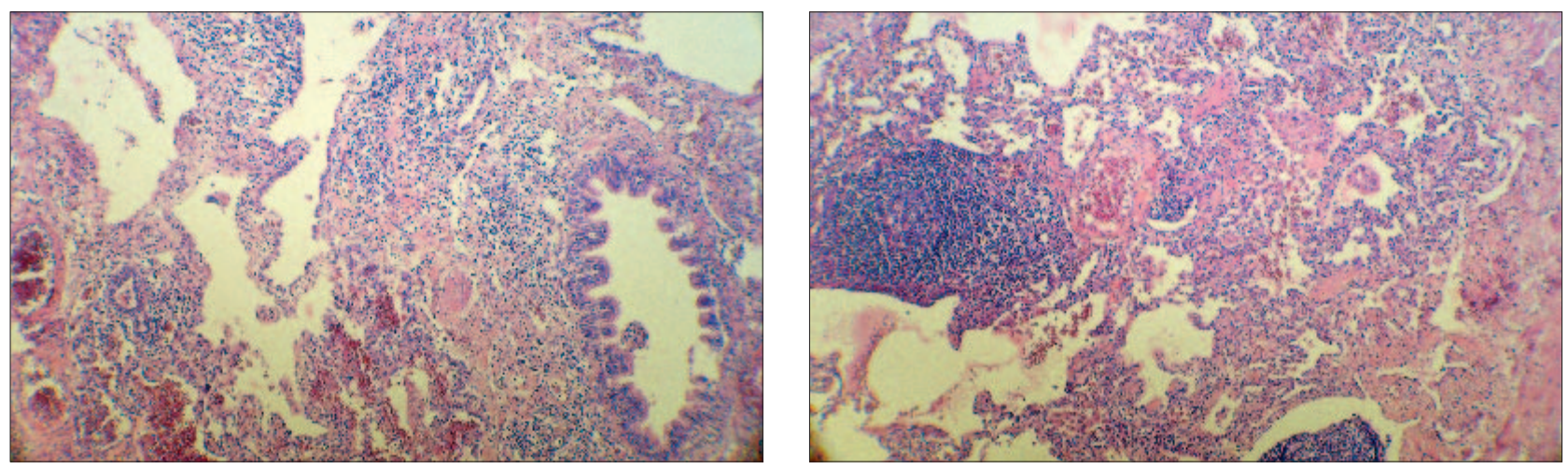

Fig. 3. Histopathological findings showing randomly distributed irregular bronchioli-like structures are separated by dilated alveolus-like structures; all are lined by cuboidal epithelial cell imparting an adenomatoid (or gland-like) appearance.

The results of laboratory tests were as follows: white blood cell count (WBC) $9,100 / / \mu \mathrm{L}$ (which increased to $18,500 / \mu \mathrm{L}$ in the next test) with $85 \%$ polymorphonuclear cells, $10 \%$ lymphocytes, and $3 \%$ monocytes. Hemoglobulin was $11.2 \mathrm{~g} / \mathrm{dL}$. Erythrocyte sedimentation rate (ESR) was 50 and C-reactive protein was $1+$. Because of hydatid cyst suspicion IgA and IgM for hydatid cyst were tested, which both were raised (14 and 1.4, respectively).

Ceftriaxone and vancomycin treatment were started in the patient at the time of admission; albendazole was also administered based on the positive IFA test and hydatid cyst suspicion.

In the chest X-ray, left-sided hyper-aeration and left lung collapse were seen (Fig. 1). In computed tomography scan, there was a multiloculated cystic lesion in left hemithorax $(172 \times 130 \times 92 \mathrm{~mm}$ in size) with mass effect and midline shift. Left lower lobe collapse was also seen (Fig. 2).

The patient underwent surgery with suspicion to hydatid cyst, but the diagnosis of adenomatoid cystic malformation was suggested in the operating room. A thoracotomy and left pneumonectomy were performed with subsequent placement of a chest tube. Finally, the diagnosis of type 3 adenomatoid cystic malformation was confirmed based on histopathological report (Fig. 3).

In follow-up, she was in good general condition without any post-surgical complaints.

\section{Discussion}

Congenital cystic adenomatoid malformation of the lung, also known as congenital pulmonary airway malformation, is a rare condition, characterized by an excessive overgrowth of the terminal bronchioles (4), which leads to enlargement of the lobe and suppression of the alveoli (5). The development of cystic lesions is controversial, but probably results from abnormal separation of localized portions of primitive tracheobronchial tree from the branching embryonic lung and a cessation of bronchomaturation and concomitant overgrowth of mesenchymal elements, which probably occur at about the 6-7th week of fetal development and which produce the adenomatoid appearances of the anomaly (6-8).

The large cyst in CCAM can lead to a mediastinal shift, which can exert pressure on the heart (5), as occurred in our case. The interesting thing in our case was her chest radiographs which sowed radiographic findings similar to hydatid cysts. This radiographic image as well as positive $\operatorname{IgA}$ and $\operatorname{IgM}$ caused the primary diagnosis of hydatic cysts in the patients.

Infants with CCAM could be asymptomatic, but some suffer from progressive respiratory distress, including tachypnea, grunting, retractions and cyanosis (3). However, our patient had none of these complaints. 
The appropriate management of congenital cystic lung disease remains a matter for debate; however, surgery seems to be the treatment of choice to remove CCAM, because of the risk of morbidity from infection, pneumothorax and even malignancy (9). Lobectomy is preferred over segmentectomy, because of the difficulty in distinguishing the extent of CCPM from normal parenchyma. However, pneumonectomy was performed in our patient, because the lesion was large and nearly all the left lung was cystic. Complications of operation for congenital lung lesions include emphysema, bronchopleural fistula, pleural effusion, and recanalization of the sequestration artery. Overall prognosis depends greatly upon the size of the lung mass and the pathophysiologic involvement. In follow-up of our patient, she had no post surgical symptoms and her general conditions were great. It should be noted that timely clinical suspicion to the disease and appropriate imaging followed by prompt surgical management are the keys to prevent further complications and even death in affected cases (9). Therefore pediatricians should be aware of such condition, while good prognosis of patients depends on timely diagnosis and good care (10). Although the management still remains controversial, early surgical excision is recommended by several authors (8).

Although routine karyotyping in all amniotic fluid obtained from the child after birth could be diagnostic, it is not cost-benefit as of extremely low incidence of chromosomal anomalies associated with CCAM (11).

In conclusion, our case demonstrates that there is a chance of misdiagnosis in patients with congenital cystic adenomatoid malformation of the lung due to radiologic similarities with hydatic cysts; therefore more careful evaluation of patients is needed to avoid such misdiagnosis.

\section{References}

1. Masters IB. Congenital airway lesions and lung disease. Pediatr Clin North Am 2009; 56: 227-242.

2. Khan NU, Jones MT, Greaves M. Case report: Congenital cystic adenomatoid malformation of an entire lung in a 33-year-old man: a case report and review of the literature. Br J Radiol 2008; 81: e276-278.

3. Sittig SE, Asay GF. Congenital cystic adenomatoid malformation in the newborn: two case studies and review of the literature. Respir Care 2000; 45: 1188-1195.

4. Al Hani HM, Al Salem AH. Congenital cystic adenomatoid malformation associated with esophageal duplication cyst. Ann Saudi Med 2005; 25: 60-62.

5. Fulghum HW, Vasquez EP. Congenital cystic adenomatoid malformation: a case study. Internet J Adv Nursing Pract 2003; 5.

6. Kravitz RM. Congenital malformations of the lung. Pediatr Clin North Am 1994; 41: 453-472.

7. Correia-Pinto J, Gonzaga S, Huang Y, Rottier R. Congenital lung lesions - underlying molecular mechanisms. Semin Pediatr Surg 2010; 19: $171-179$.

8. McDonough RJ, Niven AS, Havenstrite KA. Congenital pulmonary airway malformation: a case report and review of the literature. Respir Care 2012; 57: 302-306.

9. Andrade CF, Ferreira HP, Fischer GB. Congenital lung malformations. J Bras Pneumol 2011; 37: 259-271.

10. Masters IB. Congenital airway lesions and lung disease. Pediatr Clin North Am 2009; 56: 227-242.

11. Turan O, Hirfanoğlu IM, Beken S, Biri A, Efetürk T, Atalay Y. Prenatally detected congenital cystic adenomatoid malformation and postnatally diagnosed trisomy 13: case report and review of the literature. Turk J Pediatr 2011; 53: 337-341. 